Glasses show soft vibrational, tunnelling and relaxational modes which coexist and interact with sound waves. The fast picosecond relaxation observed in undercooled liquids seems to be due to these modes and their damping. This result is significant for theories describing undercooled liquids.

\title{
Soft Modes in Undercooled Liquids
}

The concept of normal modes requires a stable atomic structure at a local minimum of the potential energy with respect to the atomic displacements. As it successfully explains the dynamics of crystals and molecules one would think at first sight that it applies in glasses, where the atoms have fixed equilibrium positions, but does not apply in liquids, where the atoms diffuse away. It turns out that this is not the main difficulty. The diffusive motion is strongly temperature-dependent; by reducing the temperature, one can always achieve a situation where the diffusive motion is negligible within many vibrational periods, while the temperature is still well above the glass transition temperature $T_{\mathrm{g}}$.

The main obstacle for the straightforward application of the normal mode concept to disordered matter is in fact found in the glass itself where there is convincing experimental evidence [1] for tunnelling modes. Though their number is small, of the order of $10^{-5}$ tunnelling states per atom, their existence implies that one has to deal with a multi-minimum situation in the potential energy, with small energy barriers between the different minima. If one insists on an expansion of the potential energy in powers of atomic displacements, such a situation only becomes stable by taking at least fourth-order terms into account.

Within the last decade, this principal difficulty has been addressed with some success by the phenomenological soft-potential model [2], an extension of the well-known tunnelling model to include soft vibrations. The model starts from the coexistence of sound waves and localised modes. The localised modes are assumed to have small force constants which can be positive or negative, but are stabilised by a positive fourth-order term. The model is supported by findings in glasses at low temperatures.

\section{Glasses at Low Temperatures}

While the low-temperature properties of glasses cannot be understood in terms of the standard Debye model, they seem to be nonetheless universal [1]. Below $1 \mathrm{~K}$, one finds a linear specific heat and the thermal conductivity increases as $T^{2}$. These features, as well as the unusual low-temperature behaviour of the sound velocity and

Ulrich Buchenau is a senior scientist with the Institut für Festkörperforschung. Forschungszentrum Jülich, Postfach 1913, W-5170 Jülich, and is also affiliated to the University of Düsseldorf, where he became a professor in 1984 Acting Director of the Institute in 1989-90, Professor Buchenau studied at the University of Munich, receiving his Ph.D. in 1968 before working at the TU Braunschweig for six years.

\section{U. Buchenau}

\author{
Institut für Festkörperforschung, Forschungszentrum \\ Jülich, Germany
}

absorption, can be explained by the tunnelling model.

Above $1 \mathrm{~K}$, one finds glassy anomalies which can no longer be accounted for by the tunnelling model. The specific heat $C_{\mathrm{p}}$ rises more strongly than the Debye $T^{3}$-term, the thermal conductivity shows a plateau, and the sound absorption increases. At still higher temperatures, there is a peak in $C_{p} /$ $T^{3}$ and a second increase of the thermal conductivity. In terms of the vibrational density of states $g(v)$ and the frequency $v$, the peak is due to a maximum in $g(v) / v^{2}$ observed universally by Raman and neutron scattering techniques.

We shall follow the custom of Raman scatterers and denote the maximum as the boson peak. It appears at a frequency at which the corresponding crystals still only have sound waves with a wavelength of the order of 10 to 20 interatomic spacings. In glasses, one finds the soft vibrational modes of the boson peak coexisting with sound waves of the same wavelength. The number of modes is two to three orders of magnitude higher than the number of tunnelling states which dominate the properties below $1 \mathrm{~K}$. Consequently, these vibrational modes are not only observed in the specific heat, but become accessible to neutron and Raman scattering as well as to numerical simulation [3].

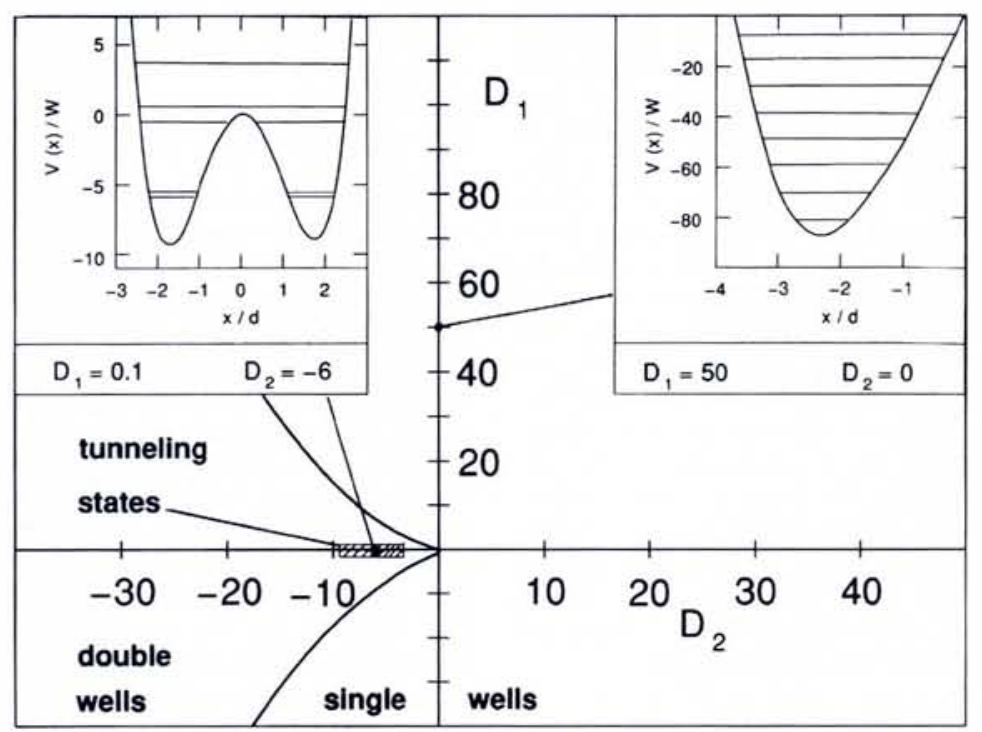

Fig. 1 - Single-and double-well regions in the $D_{1}-D_{2}$ plane of the soft-potential model. Inserts: potentials and levels of a typical tunnelling state (left) and a typical vibrational state (right).

\section{Soft-potential model}

It has recently been shown that all of these glassy anomalies can be described consistently by the soft-potential model. The model postulates soft localised modes with an effective mass $M$ and a stabilising fourthorder term in the potential

$$
V(x)=W\left[D_{1}(x / d)+D_{2}(x / d)^{2}+(x / d)^{4}\right] .
$$

The origin of the configurational coordinate $x$ is chosen such that the third-order term of the potential vanishes. The coefficients $D_{1}$ and $D_{2}$ are supposed to be random with a Gaussian distribution in $D_{1}$; the distance $d$ is fixed by the condition $W=$ $h^{2} / 2 M d^{2}$ whereupon the quantum mechanical balance between potential energy and kinetic confinement energy leads to leve splittings which are greater than $W$ for any single-well potential. Smaller level splittings are only achieved in the double-well tunnelling case (see Fig. 1). Consequently, the energy $W$ marks the cross-over between tunnelling and vibrational states. In order to explain the universal anomalies in the acoustic properties and the thermal conductivity of glasses [1], both vibrational and tunnelling states are assumed to interact with the sound waves.

Fig. 1 represents the division of the $D_{1}-D_{2}$ plane into double-well and single-well regions, where the inserts show the potentials 


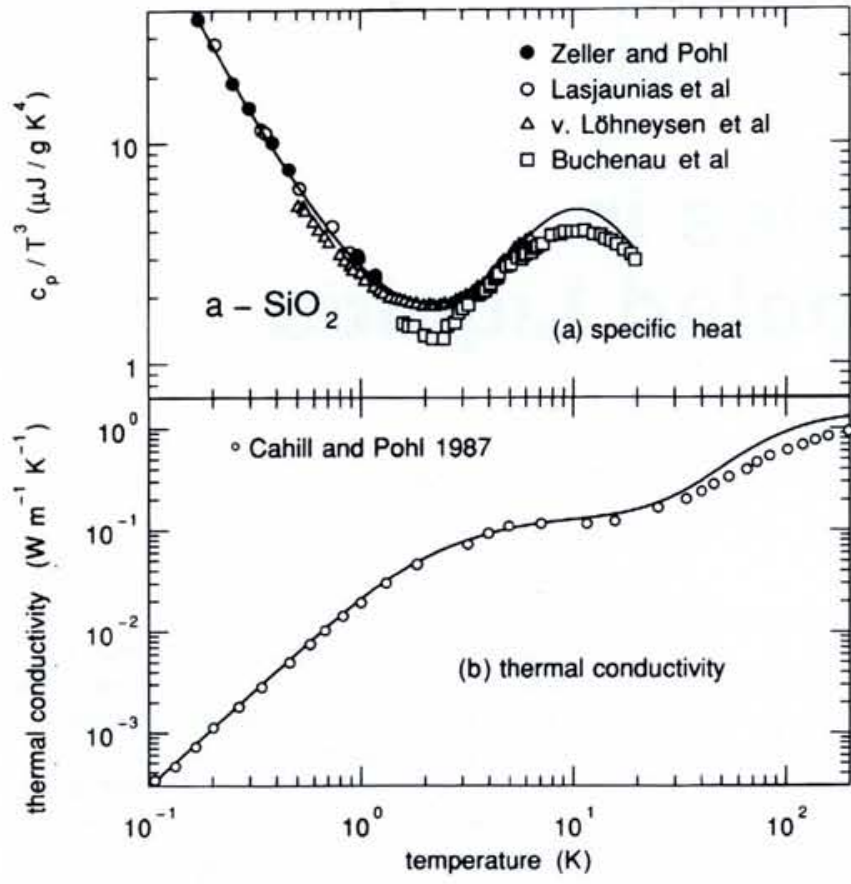

and levels of a typical tunnelling state (left) and a typical vibrational state (right). The levels were calculated using a numerical search for stationary solutions of the Schrödinger equation.

The soft-potential model allows a description of the complete low-temperature and low-frequency behaviour of the glass. As an illustration, Fig. 2 compares calculated and measured values of the specific heat, thermal conductivity and vibrational density of states data for vitreous silica [4].

\section{Barrier energy cut-off}

For the purposes of the next section, we are particularly interested in the upper cutoff of the barrier energy of the double-well potentials. This cut-off is seen in low-temperature relaxation data [5]. The best studied example is again vitreous silica where one infers a barrier cut-off at heights corresponding to 600 to $800 \mathrm{~K}$ in temperature, about one-half the glass temperature.

The existence of the cut-off supports a conceptual division of glassy relaxations into two separate groups, a low-barrier part and a high-barrier part, with the former described within the soft-potential model in terms of jumps between different wells of a soft potential. The jump eigenvectors then resemble the vibrational eigenvectors of the vibrational levels. It is obvious that these must be the most rapid structural relaxations in the glass because a vibrational level can never have a longer lifetime than its supporting atomic configuration. In addition, one also expects high-barrier relaxations, with jumps to entirely new local configurations, which can no longer be described in terms of the soft-potential model. In this case, the vibrations in the two wells should be different and the jump eigenvector should be composed of many modes of either the old or the new configurations.

\section{Johari-Goldstein relaxation}

The larger jumps should be associated with higher barriers and much longer relaxa-

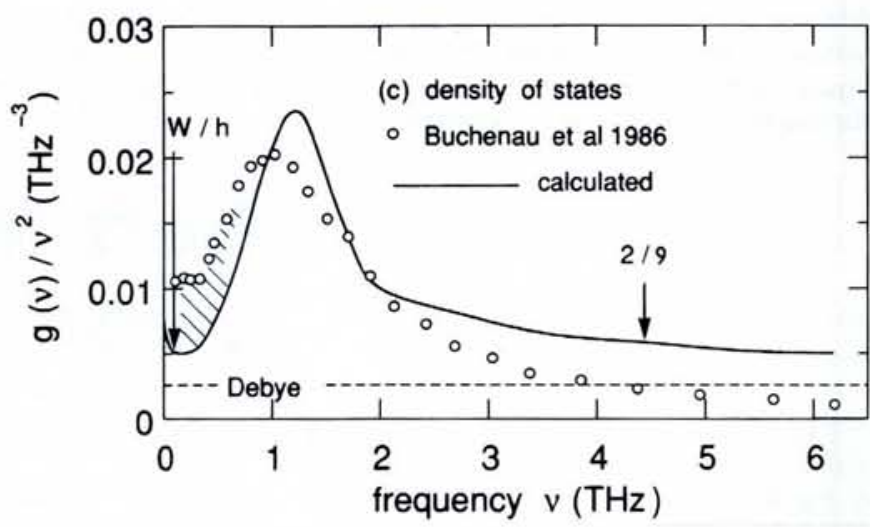

Fig. 2 - Comparison of the soft-potential model calculations [4] (continuous lines) with experimental data for vitreous silica: (a, upper left) specific heat plotted as $C_{p} / T^{3}$ versus $T$; (b,lower left) thermal conductivity versus $T ;\left(c\right.$, right) density of states, plotted as $g(v) / v^{2}$ versus frequency $\mathrm{v}$.

tion times than the low-barrier, soft-potential ones. In fact, towards higher temperatures, as one approaches $T_{\text {g }}$, an increase in the acoustic absorption is usually observed once again. It resembles a precursor to the strong $\alpha$-relaxation, setting in at $T_{\mathrm{g}}$ and connected to the flow processes. This extended tail of the $\alpha$-relaxation into the glass phase also seems to be universal [6]

Though originally denoted as $\beta$-relaxation, we shall denote it here as Johari-Goldstein relaxation in order to distinguish it from the fast picosecond relaxation in the undercooled liquid. The Johari-Goldstein relaxation shows an Arrhenius behaviour with barrier heights of the order of $20 k_{\mathrm{B}} T_{\mathrm{g}}$, where $k_{\mathrm{B}}$ is Boltzmann's constant, nearly two orders of magnitude larger than the upper cut-off of the barriers of the soft-potential model. Thus, while a fast soft-potential model relaxation occurs within a given glass configuration, with jump eigenvectors which resemble the vibrational eigenvector of the vibration within one of the wells, the slow Johari-Goldstein relaxation must be a jump to an entirely new local configuration. In the next section, we will carry over this distinction into the undercooled liquid.

\section{Consequences for the Liquid}

We have seen that there appears to be a large number of soft anharmonic localised vibrational modes in glasses, coexisting with the sound waves of wavelengths larger than ten interatomic spacings and giving rise to the boson peak in neutron and Raman scattering. These modes do not exist in textbook crystals and must be an intrinsic feature of the undercooled liquid, freezing in at the glass transition. In view of the large atomic amplitudes and the strong anharmonicity of these modes, the question arises whether they should not be overdamped in the undercooled liquid. However, as long as the temperature remains in the neighbourhood of $T_{\mathrm{g}}$, this seems not to be the case.

Neutron and Raman scattering data at temperatures 50 to $60 \mathrm{~K}$ above the glass transition in different systems still show a boson peak, clearly at a non-zero frequency. If these modes were overdamped, one would only see a broad quasielastic scattering centred around zero frequency, as is indeed observed at still higher temperatures. Fig. 3 shows this behaviour in neutron data from glassy and liquid selenium with $T_{g}$ $=305 \mathrm{~K}$. The figure displays the results in terms of a quasiharmonic density of states $g(v)$, plotted as $g(v) / v^{2}$, which apart from minor corrections, is the scattering function divided by the temperature. In a perfect harmonic system, this quantity should be independent of the temperature. In fact, there is not a large difference between the results at 100 and $300 \mathrm{~K}$. Both show the Bose peak in reasonable agreement with a soft-potentia model fit. At $360 \mathrm{~K}$, some $55 \mathrm{~K}$ above $T_{\text {g, }}$, the boson peak is still seen as a separate peak although at a lower frequency. Apart from the softening, there appears to be an increase in the number of soft modes with increasing temperature. At $440 \mathrm{~K}$, there is

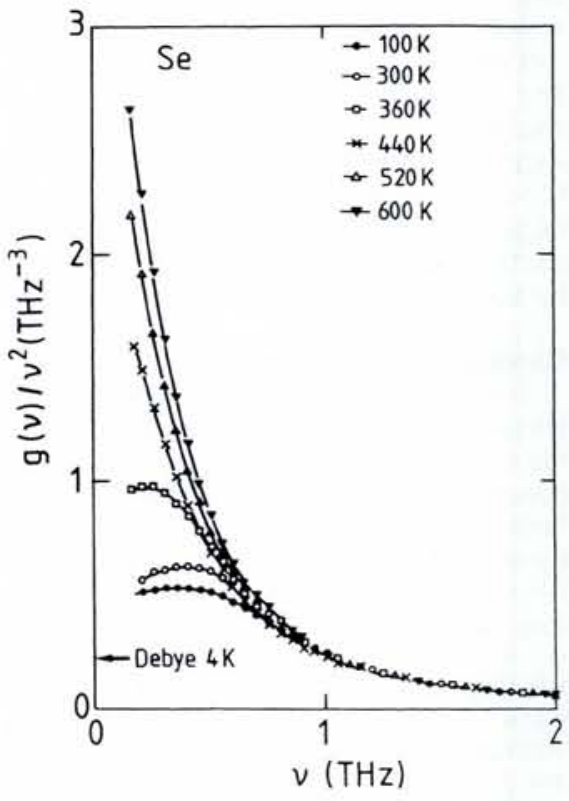

Fig. 3 - Quasiharmonic vibrational density of states fitted to neutron data in glassy and liquid selenium below and above the glass temperature $T_{g}=305 \mathrm{~K}$. 
no longer a separate boson peak and the scattering looks quasielastic, as expected for overdamped modes.

\section{Scattering from anharmonic modes}

If one assumes the soft-potential model to be a valid description, there should be an important difference between the scattering from these soft modes and from crystalline vibrations. The difference concerns the quasielastic scattering connected with the damping of the modes. For the crystalline modes, vibrating in a reasonably harmonic potential, there is practically no quasielastic scattering; this is not the case for strongly anharmonic modes.

The difference has been quantified in a recent theoretical treatment of the scattering from one-dimensional potentials by Condat and Jäckle [7]. They showed that the meansquare displacement of an anharmonic mode may be divided into two parts: the first stems from a properly weighted average over the possible vibrational levels of the potential and it leads to a reduction of the elastic intensity. The missing intensity appears in the inelastic scattering, at the vibrational frequencies and their harmonics, well separated from the elastic line. The second part of the mean-square displacement originates from transitions between different vibrational levels; it appears only if the leve change is accompanied by a displacement. It is therefore absent in symmetric singlewell potentials such as, for instance, the harmonic potential. It is, however, a familiar property for the case of classical Arrhenius relaxation over the potential barrier in a double-well potential, though one usually does not think about this case in terms of a mean-square displacement connected with the relaxational jumps.

\section{Quasielastic scattering}

In Condat and Jäckle's generalisation, the anharmonic single-well potential of the righthand insert of Fig. 1 also has a relaxational component of this type in its mean-square displacement. In fact, one can see that the centre of gravity of these energy levels shifts to the right with increasing level energy. Thus, one has a kind of localised diffusional motion as the system samples the different energy levels. Again, this second part of the mean-square displacement reduces the elastic intensity, but now the missing intensity is not found to be well-separated from the elastic line, but as quasielastic scattering centred around the elastic line. The width of the quasielastic line depends on the lifetime of the levels. If this is ten vibrational periods, the line width will be about one-tenth of the vibrational frequency.

\section{Summary}

To summarise, the soft-potential model postulates the existence of a fast quasielastic scattering component, increasing with the square of the momentum transfer and comparable in intensity to the boson peak. As the temperature increases, the width of the quasielastic scattering should increase until it merges with the boson peak. If one interprets the neutron and Raman measurements in different liquids in terms of this model, the soft modes become
Fig. 4 - Mean-square displacements in glassy, liquid and crystalline selenium determined from neutron data for motions with frequencies above $10^{11} \mathrm{~Hz}$. The quantity $\left\langle u^{2}\right\rangle_{l o c}$ is the meansquare displacement due to the localised soft modes.

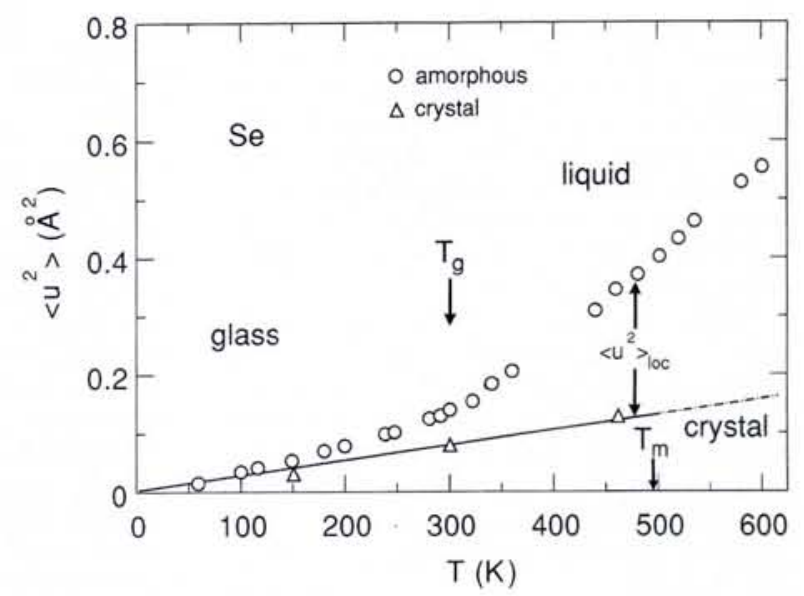

overdamped at $T \approx 1.2-1.3 T_{\mathrm{g}}$, in the close neighbourhood of the critical temperature $T_{c}$ of the mode-coupling theory which represents one among several theories for the undercooled liquid.

The fast relaxation of the damped soft modes is conceptually different from the slower structural relaxation of the flow process. The fast relaxation happens within a given glass configuration, while the slower structural relaxation requires a jump from one glass configuration to another (neighbouring) one in phase space. This conceptual difference should persist up to a temperature where the slower structural relaxation becomes so rapid that it enters the picosecond time-scale of the fast relaxation. While varying from substance to substance, this temperature on the whole tends to be rather high (of the order of $2 T_{\mathrm{g}}$ ).

\section{Relation to Other Approaches}

Since the undercooled liquid is poorly understood, there are several competing approaches for explaining experimental observations. The situation has been reviewed recently by Jäckle [8]. We shall discuss here the relation between some of these approaches and the model involving damped soft modes.

We begin with the connection to a closely related idea, namely the theory of instantaneous normal modes [9] used to interpret molecular dynamics calculations of liquids. It is based on the calculation of the dynamical matrix for the instantaneous atomic positions where one finds not only positive eigenvalues, i.e., stable modes, but also negative eigenvalues corresponding to unstable modes. The higher the temperature and the lower the density, the larger the fraction of unstable modes. Modes with either slightly positive or negative eigenvalues should be identical with the soft modes of the soft-potential model.

From the results of these instantaneous normal mode calculations one would indeed expect an increase of the number of soft modes with increasing temperature, as postulated in the preceding section to explain the rise in the quasiharmonic density of states at the boson peak between 300 and $360 \mathrm{~K}$ in Fig. 3. Such calculations should, in principle, be able to provide a much more detailed picture of these soft modes and their damping than the one we have now.
However, nobody has been able to determine the localization or the damping of the instantaneous normal modes, or to extract their stabilising fourth-order term.

We turn next to the connection between the soft-mode model and the traditional freevolume approach [8]. The latter ascribes the flow process to fluctuating free volume, which allows an atom to make a jump if enough free volume is present in its immediate neighbourhood to form a vacancy. The free volume is thought to be responsible for the larger thermal expansion of the undercooled liquid as compared to the glass. It is postulated to extrapolate to zero at the nonzero Vogel-Fulcher temperature below $T_{\mathrm{g}}$. The scheme is closely related to Kauzmann's idea of a structural entropy extrapolating to zero at the non-zero Kauzmann temperature. If one accepts its assumptions, one can derive the empirical Vogel-Fulcher law for the viscosity, according to which the viscosity would diverge at the Vogel-Fulcher temperature.

The connection between the free-volume approach and the soft-mode model has for the case of selenium. The free volume was identified with the $\left\langle u^{2}\right\rangle_{\text {loc }}$ of the meansquare displacement due to the localised soft modes. The reason for this identification is seen from Fig. 4 showing the mean-square displacements of glassy, liquid and crystalline selenium as a function of $T$. The additional mean-square displacement $\left\langle u^{2}\right\rangle_{\text {loc }}$ of the soft modes extrapolates to zero at a non-zero temperature below $T_{\mathrm{g}}$. In fact, if, in the spirit of the derivation of the VogelFulcher law from the free-volume idea, one postulates a relation between the viscosity and $\left\langle u^{2}\right\rangle_{\text {loc }}$, one obtains a much better fit for the viscosity of selenium than from the Vogel-Fulcher law.

The relation to Angell's classification [8] of strong or fragile undercooled liquids is supplied by the connection between the classification scheme and the free-volume approach. In the latter, fragile glasses are able to closely approach the Vogel-Fulcher temperature, where the free volume vanishes, while still being above $T_{\mathrm{g}}$. They therefore have only little free volume left in the glassy phase, which means that an Arrhenius plot of the viscosity is strongly curved. In the soft-potential model, this means that the number of soft modes becomes comparabeen discussed in detail recently [10], again 
tively small, as can indeed be seen at low temperatures. In vitreous silica, a strong glass, the boson peak amplitude is six to seven times larger than the signal from the sound waves, while the amplitude in the more fragile selenium is approximately the same size as the sound wave signal; the same result can be inferred from the height of the peak in $C_{\mathrm{p}} / T^{3}$ in Fig. $2 \mathrm{a}$.

Finally, we come to the connection between the soft-potential model and the mode-coupling theory [11] based on a successful treatment of the fast picosecond motion in simple liquids. The theory explains the rapid rise of the viscosity with decreasing temperature in terms of a simplified equation of motion for the density correlation function of the liquid. This equation leads again to a divergence of the viscosity, only this time at a critical temperature $T_{c}$ above $T_{g}$. The result implies that the fast picosecond relaxations of the mode-coupling theory should suffice in describing the flow process, and that the high-barrier processes need not be invoked, at least above $T_{c}$. So the question is whether these fast relaxations have anything to do with the soft modes of the soft-potential model in the same frequency region. If they have, then one could hope for a detailed microscopic basis for the mode-coupling theory in a specific substance.

\section{Conclusions}

The successful description of the lowtemperature anomalies of glasses in terms of the empirical soft-potential model implies the existence of soft anharmonic localised modes in undercooled liquids. In agreement with the results of numerical work, the number of such modes seems to increase with increasing temperature above the glass transition, and their damping gives rise to a fast quasielastic component in neutron and light scattering data.

The experimental identification of a nearly temperature-independent width of the quasielastic scattering at higher temperatures can be explained by assuming that the anharmonic modes become overdamped about $100 \mathrm{~K}$ above $T_{g}$, in the close neighbourhood of the critical temperature of the mode-coupling theory.

[1] Phillips W.A., Ed., Amorphous Solids: Low-Temperature Properties (Springer-Verlag, Berlin) 1981.

[2] Karpov V.G., Klinger M.I. \& Ignat'ev F.N., Zh. Eksp. Teor. Fiz. 84 (1983) 760 (1983); Sov. Phys. JETP 57 (1983) 439.

[3] Schober H.R. \& and Laird B.B., Phys. Rev. B 44 (1991) 6746.

[4] Gil L., Ramos M.A., Bringer A. \& Buchenau U., Phys. Rev. Lett. 70 (1993) 182.

[5] Hunklinger S. \& von Schickfus M., in [1] p. 81.

[6] Johari G.P. \& Goldstein M., J. Chem. Phys. 53 (1970) 2372.

[7] Condat C.A. \& Jäckle J., Phys. Rev. B 46 (1992) 8154.

[8] Jäckle J., Rep. Prog. Phys. 49 (1986) 171. [9] Buchner M., Ladanyi B. \& Stratt R.M., J. Chem. Phys. 97 (1992) 8522.

[10] Buchenau U. \& Zorn R., Europhys. Lett. 18 (1992) 523.

[11] Bengtzelius U., Götze W. \& Sjölander A., J. Phys. C 17 (1984) 5915.

\section{Europhysics Notes}

\section{- Announcements Delayed}

Owing to delays on agreement being reached between the Commission of the EC and selection decisions (in April) by the Programme Committee of the Human Capital and Mobility (HCM) Programme, formal announcements of the results of the latest selection rounds will probably not be made before June.

E-mail fellowship lists: EPS makes the lists of Selected Installations, Fellowship institutes, and Networks available by e-mail (simply send a message to epnews @ cernvm.cern.ch) as useful information on the availability of $\mathrm{HCM}$ fellowships can be obtained from contacts and coordinators. Such lists were released in Oct. 1992 (Installations and Fellowship institutes: 1st round), Jan. 1993 (Networks; 1st round, 1st list), May 1993 (Networks: 1st round, 2nd list). The delay affects publishing lists for:

- Installations: 2nd round pre-selection (47 pre-selected; final selection in Sept. 1993).

- Institutional fellowships: 2 nd round (678)

- Individual fellowships: 4th round

- Networks: 2nd round (62)

Euroconferences: 2nd round $(\approx 60)$.

Closing dates for future selection rounds in the HCM programme: Installations (no more); Institutional fellowships: early-1994; Individual fellowships: 15 July 1993, 1994; Networks: 15 June 1993, end-1993; Euroconferences: 15 June 1993, Oct. 1993.

\section{- NuPECC Recommends Radioactive Beams R\&D Network}

A Nuclear Physics European Collaboration Committee (NuPECC) Study Group chaired by R.H. Siessem has issued its report of the physics case and technical options for accelerated radioactive beams (ARB's) which are expected to become a major tool for nuclear structure physics as "they will greatly extend the opportunities available for the discovery of novel phenomena in nuclei far from stability". The report recommends that an R\&D network be established to ensure that an ultra high-intensity European ARB facility be built at the start of the next millennium. R\&D projects on very high-power positive ion sources are vital so that full use can be made of existing high-intensity primary beams employed in the spallation approach. Concerning other regions of bombarding energies and nuclear species, development of CERN's ISOLDE facility for very low energies should be continued; the ARENAS facility for nuclear astrophysics which is being upgraded should be made accessible to European physicists - as should a facility proposed by GANIL for extreme $N / Z$ values and at high energy. Finally, NuPECC endorses the EXCYT project calling for the development of negative ion sources for a precision tandem beam.

P. Kienle, the Chairman of NuPECC, reports that the committee decided at its meeting on 7-9 May in Madrid to assess the implications and applications of nuclear science to other fields. Working groups will point out opportunities and perspectives for some 10 fields of science and applications in a very ambitious and interdisciplinary study that will hopefully come up with concrete proposals for presentation to governments.
Another NuPECC topic of high-priority is a future continuous-wave electron accelerator. The European Electron Accelerator Project [EN 23 (1992) 136] with a four-country steering committee plans to issue its report in Sept./ Oct. for presentation to funding agencies.

\section{Meetings}

15-21 Aug. 1993 Neutron Scattering: Summer School Zuoz, Switzerland

R. Bercher, PSI, CH-5232 Villigen PSI

tel./fax: +41 (56) 993402 / 982327

A: 30 Jun $93 / \lim 100 /$ SFR 420.-; incl. board

22-27 Sept. 1994 Thinking Science for Teaching - The Case for Physics: Int. Conf. Rome, Italy P. Maiolo, Lab. di Didattica delle Scienze, Dip. Fisica, Univ. "La Sapienza", P.le Aldo Moro, 2, 1-00185 Rome Ab: 30 Nov 93 / PP: 15 Jun 94 / lim $100 /$ SUS 100 .

\section{Corrections to March 1993 List}

Conference: undecided 1994 (not School: Varenna, 25 Aug. - Sept. 2, 1993) Adv. Toroidal Devices with Innovative Physics \& Technolgy Varenna, Italy E. Sidoni, ISSP, Via Celoria, 16, I-20133 Milan

tel./fax: +39 (2) $2392267 / 2392205$

6-14 Sept. 1994 (not 1993) Physics \& Technology of Tritium in Fusion Reactors: Course \& Workshop Varenna, Italy E. Sidoni, ISSP, Via Celoria, 16, I-20133 Milan tel./fax: +39 (2) 23922 67/239 2205

\section{Technological University of Delft} (The Netherlands)

The Faculty of Applied Physics has an opening for a position of

\section{Full Professor in COMPUTATIONAL PHYSICS}

The duties involve teaching of physics and research activity in computational physics, i.e., the use and development of modern simulation techniques, numerical techniques and, if necessary, special hardware processors, aimed at the study of microscopic (many body) and macroscopic (wave propagation and flow) physical phenomena. The applicant is expected to pursue cooperation with and give support to the research of other groups both concerning macroscopic and microscopic problems.

The candidate should be a creative physicist with experience in simulation techniques of physical (microscopic and/or macroscopic, processes, with a good knowledge of hardware and software, and with a clear interest in the development of simula. tion systems. $\mathrm{He}$ or she should have an international reputation and be an experienced and inspiring teacher with proven didactic talent.

Further information about this position can be obtained from prof.dr.ir. A.J. Berkhout, telephone +3115 781804 .

Applications, including a curriculum vitae, a list of publications, and at least two letters of reference, should be submitted within three weeks of the appearance of this announcement to: Faculteit der Technische Natuurkunde, Dienst Personeel en Organisatie, Lorentzweg 1, NL-2628 CJ Delft, The Netherlands. Please quote REF: TN 9315.

The University follows a policy of equal opportunity in employment and invites both qualified women and qualified men to apply for this post. 